\title{
Finite Element Analysis of Thermal-Diffusions Problem for Unbounded Elastic Medium Containing Spherical Cavity under DPL Model
}

\author{
Aatef D. Hobiny ${ }^{1}$ (D) and Ibrahim A. Abbas $1,2, *(\mathbb{D})$ \\ 1 Department of Mathematics, Faculty of Science, King Abdulaziz University, Jeddah 21589, Saudi Arabia; \\ ahobany@kau.edu.sa \\ 2 Mathematics Department, Faculty of Science, Sohag University, Sohag 82524, Egypt \\ * Correspondence: ibrabbas7@science.sohag.edu.eg or aabbas5@kau.edu.sa
}

check for updates

Citation: Hobiny, A.D.; Abbas, I.A. Finite Element Analysis of

Thermal-Diffusions Problem for

Unbounded Elastic Medium

Containing Spherical Cavity under DPL Model. Mathematics 2021, 9, 2782. https://doi.org/10.3390/ math9212782

Academic Editors: Marin Marin, Nicolae Pop and Sorin Vlase

Received: 6 October 2021

Accepted: 27 October 2021

Published: 2 November 2021

Publisher's Note: MDPI stays neutral with regard to jurisdictional claims in published maps and institutional affiliations.

Copyright: (c) 2021 by the authors. Licensee MDPI, Basel, Switzerland. This article is an open access article distributed under the terms and conditions of the Creative Commons Attribution (CC BY) license (https:/ / creativecommons.org/licenses/by/ $4.0 /)$.

\begin{abstract}
In this work, the thermo-diffusions interaction in an unbounded material with spherical cavities in the context dual phase lag model is investigated. The finite element technique has been used to solve the problem. The bounding surface of the inner hole is loaded thermally by external heat flux and is traction-free. The delay times caused in the microstructural interactions, the requirement for thermal physics to take account of hyperbolic effects within the medium, and the phase lags of chemical potential and diffusing mass flux vector are interpreted. A comparison is made in the case of the presence and the absence of mass diffusions between coupled, Lord-Shulman and dual phase lag theories. The numerical results for the displacement, concentration, temperature, chemical potential and stress are presented numerically and graphically.
\end{abstract}

Keywords: finite element analysis; dual phase lag model; thermo-diffusions

\section{Introduction}

The classical thermoelastic theory [1] depends on the Fourier hypothesis of heating conductivity. Biot [2] presented the solution to this paradox by introducing the new idea of the coupled thermoelastic model. Diffusions can be defined as the random process of a set of particles moving from the region of highest concentration to a region of lower concentration. Generalized thermoelasticity, a new milestone in the various theories of thermo-elasticity, prevails over the significant imperfections of classical theories of thermoelasticity. Lord and Shulman [3] are the two pioneers who contributed most to coupled thermo-elasticity by introducing the generalized theory of thermo-elasticity through alteration of the parabolic nature of the heat conduction equation to a hyperbolic nature. They made this revolutionary change in the nature of the heat conduction equation by incorporating a relaxation time parameter in Fourier's law of heat conduction and, in doing so, the unrealistic phenomenon of the infinite speed of thermal wave propagation was replaced by practical observation of the finite speed of propagation of thermal waves. The study of diffusions became increasingly important, mainly due to its multiple application in industrial applications and geophysics. In integrated circuit manufacturing, diffusions are used to introduce "dopants" in controlled quantities in the semi-conductor substrates. First of all, the theory of thermo-elasticity arose during the discussion of the coupled and decoupled theory, which has no compatibility with physical experiments. The theory of thermoelastic diffusion for elastically deformable solid bodies was first proposed by Nowacki [4-6], in which he derived very important corollaries, results and governing field equations for coupled thermoelastic diffusion. The coupled thermoelastic diffusion theories predict an unrealistic infinite speed of thermal wave propagation. Surpassing this impractical generalized thermoelastic diffusion theory, the Lord-Shulman model was first proposed by Sherief, et al. [7], introducing a diffusion relaxation parameter into the well-known 
Fick's law of mass diffusion. The dual-phase lag theory of thermal conduction has been proposed by Tzou [8]. Abbas and Seingh [9] used the finite element method to investigate the effects of rotating thermo-elastic half-space with diffusion. Villani, Aurélien, et al. [10] studied the numerical implementation, analytical validation, and the effects of plasticity on equilibrium for a fully coupled diffusional-mechanical formulation. Abbas et al. [11] discussed the responses of thermal sources in a transversely isotropic thermo-elastic plane with mass diffusion by finite element schemes. Sharma et al. [12] studied the thermodiffusion interaction in an unbounded medium with a cylindrical hole. Abbas and Marin [13] studied the analytical solution of a 2D generalized thermoelastic diffusion problem caused by the laser pulse. Abouelregal et al. [14] studied fractional thermo-elastic diffusion models with four thermal delay times. The vibrations of an orthotropic double-layered graphene sheet upon the hygrothermal condition were studied by Sobhy using the trigonometric shear deformations plate model [15]. Othman and Eraki [16] have discussed generalized magneto-thermo-elastic diffusion in a half-space under initial stress. Lotfy [17] investigated the effects of variable thermal conductivity through the photothermal diffusion processes of semi-conductor material. Kumar et al. [18] studied the effects of thermal and diffusion phase-lags in a plate with axisymmetric heat supply. Abbas and Kumar [19] used the finite element method to study the deformations caused by the thermal sources in a micropolar generalized thermoelastic plane. Bezzina and Zenkour [20] studied the thermo-elastic diffusions of a solid cylinder under the modified Green-Naghdi model. Madureira et al. [21] presented numerical analysis for thermo-diffusion problems in moving boundaries. Zenkour [22] studied the thermodiffusion of an infinite solid with a spherical hole via refined three-phase-lag GN models. Singh and Yadav [23] discussed the effects of diffusions on propagations and reflections of waves in a thermo-microstretch solid plane. Elhagary [24] investigated fractional thermoelastic diffusion problems for an infinitely long hollow cylinder under Caputo-Fabrizio definition. Molla and Mallik [25] presented the variational principles, uniqueness and reciprocity model for higher-order fractional four-phase-lag generalized thermodiffusion models. Eremeyev and Pietraszkiewicz [26] studied the nonlinear resultant theory of shells accounting for thermodiffusion. Over recent decades, several problems have been raised by generalized thermoelastic models as in [27-40].

The aim of this investigation is to introduce e dual phase lag thermo-diffusion formulations instead of Fick's classical diffusions model by using two diffusion lags. The finite element method is applied to study the thermo-diffusion in an infinite medium with a spherical cavity. The physical explanations are presented sequentially corresponding to the distribution of the considered physical variables obtained in this investigation. Numerically calculated results are represented graphically. The effects of thermal lags are shown in the different components.

\section{Mathematical Model}

Following Sherief et al. [7,18], the basic equations for an isotropic, elastic soled with the thermo-diffusions under a dual phase lag model at uniform temperature $T_{o}$ in the absence of heating sources and body forces, can be expressed as:

The equations of motion as in [7] are

$$
\mu u_{i, j j}+(\lambda+\mu) u_{j, j j}-\beta_{t} T_{, i}-\beta_{c} C_{, i}=\rho \frac{\partial^{2} u_{i}}{\partial t^{2}} .
$$

The heat conduction equation is

$$
K_{i j}\left(1+\tau_{1} \frac{\partial}{\partial t}\right) T_{, i i}=\left(\frac{\partial}{\partial t}+\tau_{2} \frac{\partial^{2}}{\partial t^{2}}\right)\left(\rho c_{e} T+\beta_{t} T_{o} e_{k k}+a T_{o} C\right) .
$$


The mass diffusion equation is

$$
D\left(1+\tau_{3} \frac{\partial}{\partial t}\right)\left(b C_{, i i}-\beta_{c} e_{k k, i i}-a T_{, i i}\right)=\left(\frac{\partial}{\partial t}+\tau_{4} \frac{\partial^{2}}{\partial t^{2}}\right) C
$$

The constitutive equations are

$$
\begin{gathered}
\sigma_{i j}=2 \mu e_{i j}+\left(\lambda e-\beta_{t}\left(T-T_{o}\right)-\beta_{c} C\right) \delta_{i j}, \\
P=-\beta_{c} e_{k k}+b C-a\left(T-T_{o}\right), \\
e_{i j}=\frac{1}{2}\left(u_{i, j}+u_{j, i}\right) .
\end{gathered}
$$

This model can be reduced to:

(i) (DPLD) refers to the dual phase lag thermo-diffusion model

$$
\tau_{1}, \tau_{2}, \tau_{3}, \tau_{4} \neq 0
$$

(ii) (LSD) refers to the Lord and Shulman thermo-diffusion model

$$
\tau_{1}=\tau_{3}=0, \tau_{2}, \tau_{4} \neq 0 .
$$

(iii) (CTD) refers to the classical thermo-diffusion model

$$
\tau_{1}=\tau_{2}=\tau_{3}=\tau_{4}=0 .
$$

where the delay time $\tau_{1}$ is interpreted as that caused by the microstructural interactions and is called the phase lag of temperature gradient, the delay time $\tau_{2}$ denotes the relaxation time required for thermal physics to take account of hyperbolic effect within the medium, $\tau_{3}$ and $\tau_{4}$ are the phase lags of chemical potential and diffusing mass flux vector, respectively, $\rho$ is the density of the material, $\lambda, \mu$ are the Lame's constants, $P$ is the chemical potential, $\sigma_{i j}$ are the components of stress tensor and $\delta_{i j}$ is the Kronecker delta symbols, $T$ is the temperature, $C$ is the concentrations of diffusion material in the elastic medium, $u_{i}$ are the displacement vector components, $\beta_{c}=(3 \lambda+2 \mu) \alpha_{c}$ and $\beta_{t}=(3 \lambda+2 \mu) \alpha_{t}, \alpha_{t}$ and $\alpha_{c}$ are the coefficients of thermal expansions and linear diffusions, respectively, $e_{k k}$ are the components of strain tensor, $c_{e}$ is the specific heat at constant strain, $K_{i j}$ is the thermal conductivity, $T_{o}$ is the reference temperature and $a$ is the measure of thermo-diffusions effect, $b$ is the measures of diffusive effects and $D$ is the coefficient of diffusions. Let us consider a homogenous and elastic unbounded medium containing a spherical cavity occupying the region $h \leq r<\infty$ as in Figure 1.

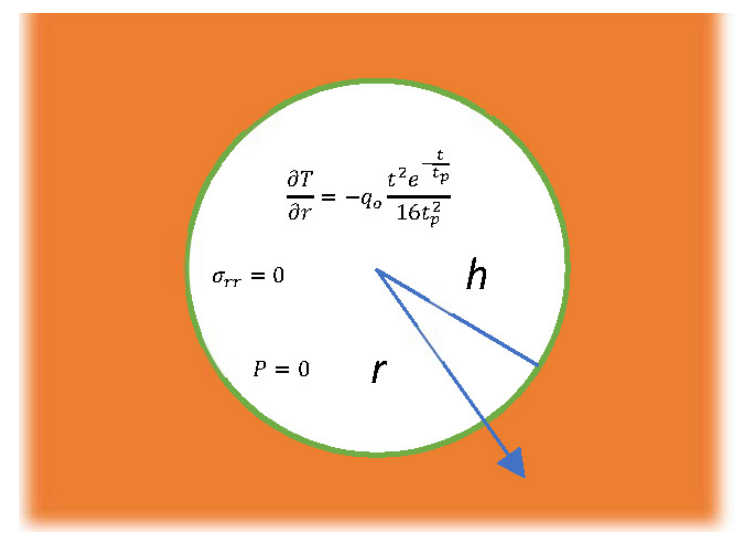

Figure 1. The sketch of an unbounded medium containing a spherical cavity. 
Due to the symmetry, all functions will be given in terms of time $t$ and the radial $r$ variable. In the system of spherical coordinate $(r, \theta, \varphi)$, the displacement components can be given by

$$
u_{r}=u(r, t), u_{\theta}=0, u_{\varphi}=0 .
$$

The non-vanishing strain components can be defined as

$$
e_{r r}=\frac{\partial u}{\partial r}, e_{\theta \theta}=\frac{u}{r}, e_{\varphi \varphi}=\frac{u}{r} .
$$

Thus, the basic equations can be written as

$$
\begin{gathered}
\sigma_{r r}=2 \mu \frac{\partial u}{\partial r}+\lambda e-\beta_{t}\left(T-T_{0}\right)-\beta_{c} C \\
\sigma_{\theta \theta}=\sigma_{\varphi \varphi}=2 \mu \frac{u}{r}+\lambda e-\beta_{t}\left(T-T_{0}\right)-\beta_{c} C \\
P=b C-\beta_{c} e-a\left(T-T_{o}\right) \\
e=\frac{\partial u}{\partial r}+\frac{2 u}{r} \\
(\lambda+2 \mu) \frac{\partial e}{\partial r}-\beta_{t} \frac{\partial T}{\partial r}-\beta_{c} \frac{\partial C}{\partial r}=\rho \frac{\partial^{2} u}{\partial t^{2}} \\
K\left(1+\tau_{1} \frac{\partial}{\partial t}\right)\left(\frac{\partial^{2} T}{\partial r^{2}}+\frac{2}{r} \frac{\partial T}{\partial r}\right)=\left(\frac{\partial}{\partial t}+\tau_{2} \frac{\partial^{2}}{\partial t^{2}}\right)\left(\rho c_{e} T+\beta_{t} T_{o} e+a T_{o} C\right), \\
D\left(1+\tau_{3} \frac{\partial}{\partial t}\right)\left(b\left(\frac{\partial^{2} C}{\partial r^{2}}+\frac{2}{r} \frac{\partial C}{\partial r}\right)-\beta_{t}\left(\frac{\partial^{2} e}{\partial r^{2}}+\frac{2}{r} \frac{\partial e}{\partial r}\right)-a\left(\frac{\partial^{2} T}{\partial r^{2}}+\frac{2}{r} \frac{\partial T}{\partial r}\right)\right)=\left(\frac{\partial}{\partial t}+\tau_{4} \frac{\partial^{2}}{\partial t^{2}}\right) C
\end{gathered}
$$

By using the following non-dimensional variables to the above equations

$$
\begin{gathered}
\left(r^{\prime}, u^{\prime}\right)=c \omega(r, u),\left(t^{\prime}, \tau_{1}^{\prime}, \tau_{2}^{\prime}, \tau_{3}^{\prime}, \tau_{4}^{\prime}, t_{p}^{\prime}\right)=c^{2} \omega\left(t, \tau_{1}, \tau_{2}, \tau_{3}, \tau_{4}, \tau_{p}\right), P^{\prime}=\frac{P}{\beta_{c}}, \\
C^{\prime}=\frac{\beta_{c} C}{(\lambda+2 \mu)}, T^{\prime}=\frac{\beta_{t}\left(T-T_{o}\right)}{(\lambda+2 \mu)},\left(\sigma_{r r}^{\prime}, \sigma_{\theta \theta}^{\prime}, \sigma_{\varphi \varphi}^{\prime}\right)=\frac{\left(\sigma_{r r}, \sigma_{\theta \theta}, \sigma_{\varphi \varphi}\right)}{(\lambda+2 \mu)}
\end{gathered}
$$

where $\omega=\frac{\rho c_{e}}{K}$ and $c=\sqrt{\frac{\lambda+2 \mu}{\rho}}$.

Using Equation (16) in Equations (9)-(15), and after that suppressing the primes, can take the forms

$$
\begin{gathered}
\sigma_{r r}=\frac{\partial u}{\partial r}+2 \alpha \frac{u}{r}-T-C \\
\sigma_{\theta \theta}=\sigma_{\varphi \varphi}=\alpha \frac{\partial u}{\partial r}+(1+\alpha) \frac{u}{r}-T-C \\
P=\zeta_{c} C-e-\zeta_{t} T \\
e=\frac{\partial u}{\partial r}+\frac{2 u}{r} \\
\frac{2}{\partial r^{2}}+\frac{2}{r} \frac{\partial u}{\partial r}-\frac{2 u}{r^{2}}-\frac{\partial T}{\partial r}-\frac{\partial C}{\partial r}=\frac{\partial^{2} u}{\partial t^{2}} \\
\left(1+\tau_{1} \frac{\partial}{\partial t}\right)\left(\frac{\partial^{2} T}{\partial r^{2}}+\frac{2}{r} \frac{\partial T}{\partial r}\right)=\left(\frac{\partial}{\partial t}+\tau_{2} \frac{\partial^{2}}{\partial t^{2}}\right)\left(T+\gamma_{t} e+\gamma_{c} C\right) \\
\left(1+\tau_{3} \frac{\partial}{\partial t}\right)\left(\zeta_{c}\left(\frac{\partial^{2} C}{\partial r^{2}}+\frac{2}{r} \frac{\partial C}{\partial r}\right)-\left(\frac{\partial^{2} e}{\partial r^{2}}+\frac{2}{r} \frac{\partial e}{\partial r}\right)-\zeta_{t}\left(\frac{\partial^{2} T}{\partial r^{2}}+\frac{2}{r} \frac{\partial T}{\partial r}\right)\right)=\zeta_{s}\left(\frac{\partial}{\partial t}+\tau_{4} \frac{\partial^{2}}{\partial t^{2}}\right) C,
\end{gathered}
$$

where $\alpha=\frac{\lambda}{(\lambda+2 \mu)}, \gamma_{t}=\frac{\beta_{t}^{2} T_{o}}{\rho c_{e}(\lambda+2 \mu)}, \gamma_{c}=\frac{a \beta_{t} T_{o}}{\rho c_{e} \beta_{c}}, \zeta_{c}=\frac{b(\lambda+2 \mu)}{\beta_{c}^{2}}, \zeta_{t}=\frac{a(\lambda+2 \mu)}{\beta_{t} \beta_{c}}, \zeta_{s}=\frac{(\lambda+2 \mu)}{D \omega \beta_{c}^{2}}$. 


\section{Initial and Boundary Conditions}

This problem can be solved subject to the initial conditions

$$
u=T=C=0, \frac{\partial u}{\partial t}=0, \frac{\partial T}{\partial t}=0, \frac{\partial C}{\partial t}=0, t=0 .
$$

The boundary conditions can be given by the inner surface of the cavity are due to heat flux with the exponentially decaying pulse [41].

$$
-\left.\frac{\partial T(r, t)}{\partial r}\right|_{r=\|}=q_{o} \frac{t^{2} e^{-\frac{t}{t_{p}}}}{16 t_{p}^{2}}, \sigma_{r r}(h, t)=0, P(h, t)=0, t>0,
$$

where $t_{p}$ is the pulse heat flux characteristic time and $q_{o}$ is a constant.

\section{Numerical Scheme}

In this section, the formulation of thermoelastic diffusion based upon thermal delay times $\tau_{1}, \tau_{2}, \tau_{3}$ and $\tau_{4}$ in an unbounded medium with spherical cavity is abbreviated by employing the finite element method (FEM). This technique is a powerful approach primarily advanced to obtain the numerical solution of a complex problem, and the finite element method is the selected method for linear/non-linear systems in several fields. The finite element scheme is applied here to obtain the solution of Formulas (19)-(21) under the initial condition (22) and the boundary (23) conditions. Abbas et al. [42-45] presented the solutions for various problems under deference generalized thermoelasticity models. The finite element formulation of thermoelastic diffusion can be obtained by using the standard procedure. The non-dimension weak formulations of dual phase lag model are derived. The set of independent test functions, to consist of the temperature $\delta T$, the displacement $\delta u$ and the concentration $\delta C$, are specified. The governing formulations are multiplied by independent weighting functions and then the boundary conditions are used to integrate over the spatial domain. The applications of integration by parts and the use of the divergence theorem to decrease the order of the spatial derivative paves the way for the application of the boundary condition. Using the Galerkin procedure, the unknown variables and the corresponding test functions are approximated by the same shape functions.

$$
\begin{gathered}
T=\sum_{j=1}^{n} N_{j} T_{j}(t), u=\sum_{j=1}^{n} N_{j} u_{j}(t), C=\sum_{j=1}^{n} N_{j} C_{j}(t), \\
\delta T=\sum_{j=1}^{n} N_{j} \delta T_{j}, \delta u=\sum_{j=1}^{n} N_{j} \delta u_{j}, \delta C=\sum_{j=1}^{n} N_{j} \delta C_{j},
\end{gathered}
$$

where $N$ points to the shape function and $n$ refers to the node numbers per element. The quadratic element with three nodes is used. In this problem, the one-dimension quadratic element is used and we suppose that the local coordinates in the range $[-1,1]$ are used for the master element which can be defined by

$$
N_{1}=\frac{1}{2}\left(\chi^{2}-\chi\right), \quad N_{1}=1-\chi^{2}, \quad N_{3}=\frac{1}{2}\left(\chi^{2}+\chi\right) .
$$

Furthermore, the unknown variables' time derivatives should be determined by the implicit approaches. Thus, the weak formulations for the finite element scheme corresponding to the basic equations can be written as

$$
\int_{h}^{L} \frac{\partial \delta T}{\partial r}\left(\frac{\partial u}{\partial r}+\frac{2 u}{r}-T-C\right) d r+\int_{h}^{L} \delta T \frac{\partial^{2} u}{\partial t^{2}} d r=\left.\delta T\left(\frac{\partial u}{\partial r}+\frac{2 u}{r}-T-C\right)\right|_{h} ^{L}
$$

$$
\int_{h}^{L} \frac{\partial \delta T}{\partial r}\left(1+\tau_{1} \frac{\partial}{\partial t}\right) \frac{\partial T}{\partial r} d r+\int_{h}^{L} \delta T\left(\left(\frac{\partial}{\partial t}+\tau_{2} \frac{\partial^{2}}{\partial t^{2}}\right)\left(T+\gamma_{t} e+\gamma_{c} C\right)-\left(\frac{\partial}{\partial t}+\tau_{1} \frac{\partial^{2}}{\partial t^{2}}\right) \frac{2}{r} \frac{\partial T}{\partial r}\right) d r=\left.\delta T\left(1+\tau_{1} \frac{\partial}{\partial t}\right) \frac{\partial T}{\partial r}\right|_{h} ^{L},
$$




$$
\begin{gathered}
\int_{h}^{L} \delta C\left(\zeta_{s}\left(\frac{\partial}{\partial t}+\tau_{4} \frac{\partial^{2}}{\partial t^{2}}\right) C-\frac{2}{r}\left(\frac{\partial}{\partial t}+\tau_{3} \frac{\partial^{2}}{\partial t^{2}}\right)\left(\zeta_{c} \frac{\partial C}{\partial r}-\frac{\partial e}{\partial r}-\zeta_{t} \frac{\partial T}{\partial r}\right)\right) d r+\int_{h}^{L} \frac{\partial \delta C}{\partial r}\left(1+\tau_{3} \frac{\partial}{\partial t}\right)\left(\zeta_{c} \frac{\partial C}{\partial r} C-\frac{\partial e}{\partial r}-\zeta_{t} \frac{\partial T}{\partial r}\right) d r= \\
\left.\delta C\left(1+\tau_{3} \frac{\partial}{\partial t}\right)\left(\zeta_{c} \frac{\partial C}{\partial r} C-\frac{\partial e}{\partial r}-\zeta_{t} \frac{\partial T}{\partial r}\right)\right|_{h} ^{L}
\end{gathered}
$$

\section{Numerical Result and Discussion}

In this part, the copper material was taken for purposes of numerical calculation and the parameters of the problem were chosen as in [46]

$$
\begin{gathered}
\rho=8954(\mathrm{~kg})\left(\mathrm{m}^{-3}\right), D=0.85 \times 10^{-8}(\mathrm{~kg})(\mathrm{s})\left(\mathrm{m}^{-3}\right), \alpha_{c}=1.98 \times 10^{-4}\left(\mathrm{~kg}^{-1}\right)\left(\mathrm{m}^{3}\right), R=1, \\
a=1.2 \times 10^{4}\left(\mathrm{~m}^{2}\right)\left(\mathrm{k}^{-1}\right)\left(\mathrm{s}^{-2}\right), b=0.9 \times 10^{6}\left(\mathrm{~m}^{5}\right)\left(\mathrm{kg}^{-1}\right)\left(\mathrm{s}^{-2}\right), \alpha_{t}=17.8 \times 10^{-6}\left(\mathrm{k}^{-1}\right), \\
\mathcal{c}_{e}=383.1\left(\mathrm{~m}^{2}\right)\left(\mathrm{s}^{-2}\right)\left(\mathrm{k}^{-1}\right), K=3.68 \times 10^{2}(\mathrm{~kg})\left(\mathrm{s}^{-3}\right)\left(\mathrm{k}^{-1}\right)(\mathrm{m}), T_{o}=293(\mathrm{k}), T_{1}=1, \\
\mu=3.86 \times 10^{10}(\mathrm{~kg})\left(\mathrm{m}^{-1}\right)\left(\mathrm{s}^{-2}\right), \lambda=7.76 \times 10^{10}(\mathrm{~kg})\left(\mathrm{m}^{-1}\right)\left(\mathrm{s}^{-2}\right) .
\end{gathered}
$$

The computations are performed for the estimations of time $(t=0.2)$, presence and absence of different time delay parameters. The finite element method is employed to obtain the natures of the radial displacement, the temperature, the concentration, the chemical potential with the radial and the shear stresses distribution with respect to the radial distance $r$. The grid size has been refined until the values of considered fields stabilize. Further refinement of mesh size over 20,000 elements does not change the values considerably. Thus, the grid size 20,000 has been used for this study. Figure 2 shows the variation of radial displacement along the distance $r$. It is noted that the radial displacement has maximum negative values at the internal surface of hole $r=h$. The magnitude of radial displacement increases from the negative values to positive values. In these values, the radial displacements have peak values that depend on the type of model. Figure 3 represents the variations of temperature with respect to the distance $r$. It is observed that it always starts from the difference of certain values according to the values of heat flux with the increasing of the radial distance to reach zero values. Figure 4 represents the deviation of radial stress with respect to the radial distance $r$ for different models. It is easy to see the radial stress decreases from zero which satisfy the problem boundary conditions to maximum negative values, and after that increases quickly as the radial distance $r$ increases, then the stress decreases to zero. Figure 5 displays the variations of hoop stress versus the radial distance $r$ for three different models. It is observed that the magnitude of hoop stress starts from maximum values which depend on the type of model, then increases with the increase of the distance $r$ to close to zero. Figure 6 displays the distribution of concentration versus the radial distance $r$. It is clear that the concentration has maximum values at the internal surface of the cavity after which it decreases with the increase of distance $r$. Figure 7 shows the variations of chemical potential versus the redial distances. From Figure 6, it is observed that the chemical potential begins from zero value, which agrees with the boundary condition, and increases with the rising of the radial $r$. The chemical potential has peak values that depend on the value of the type of model. 


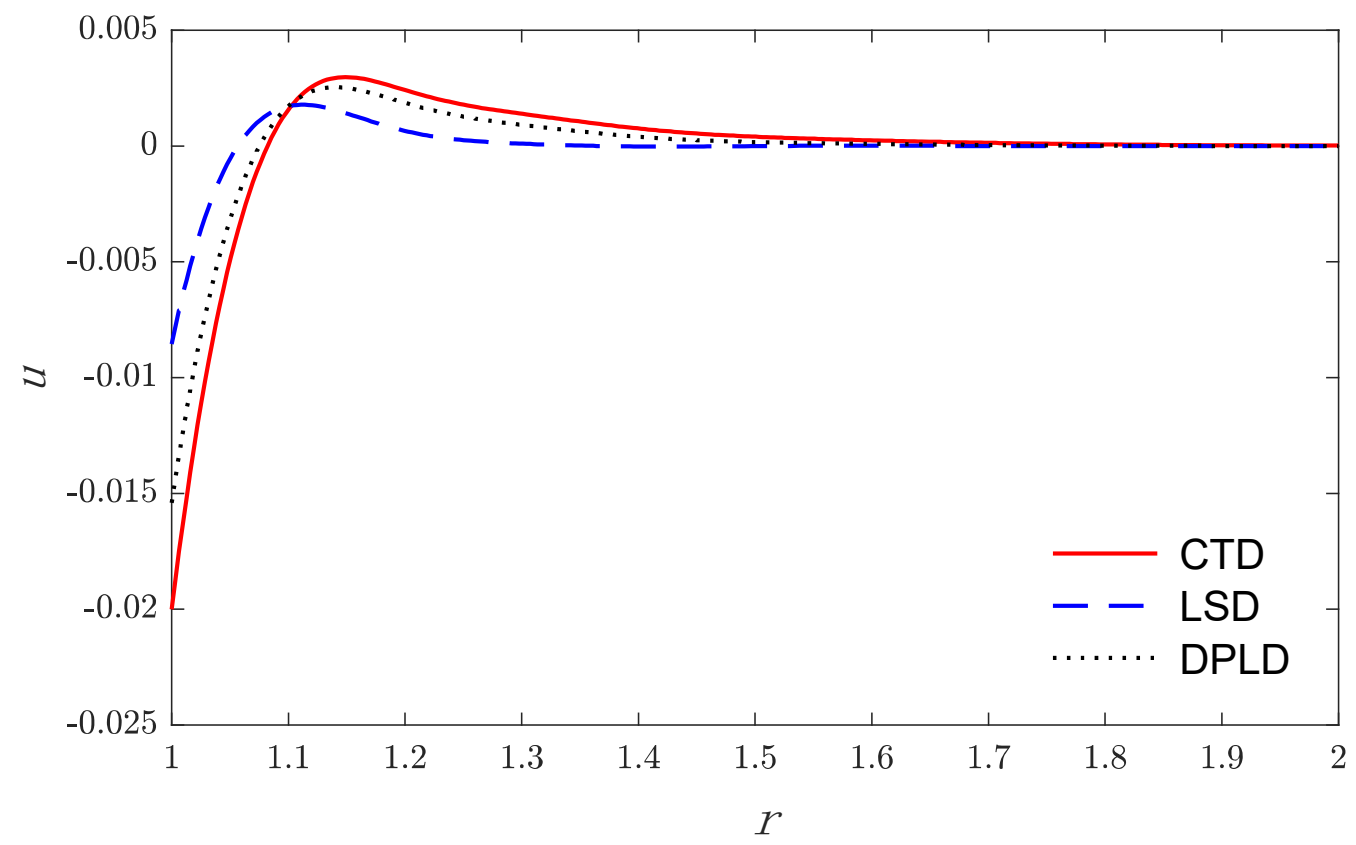

Figure 2. The redial displacement variations $u$ versus the redial distance $r$ for three models.

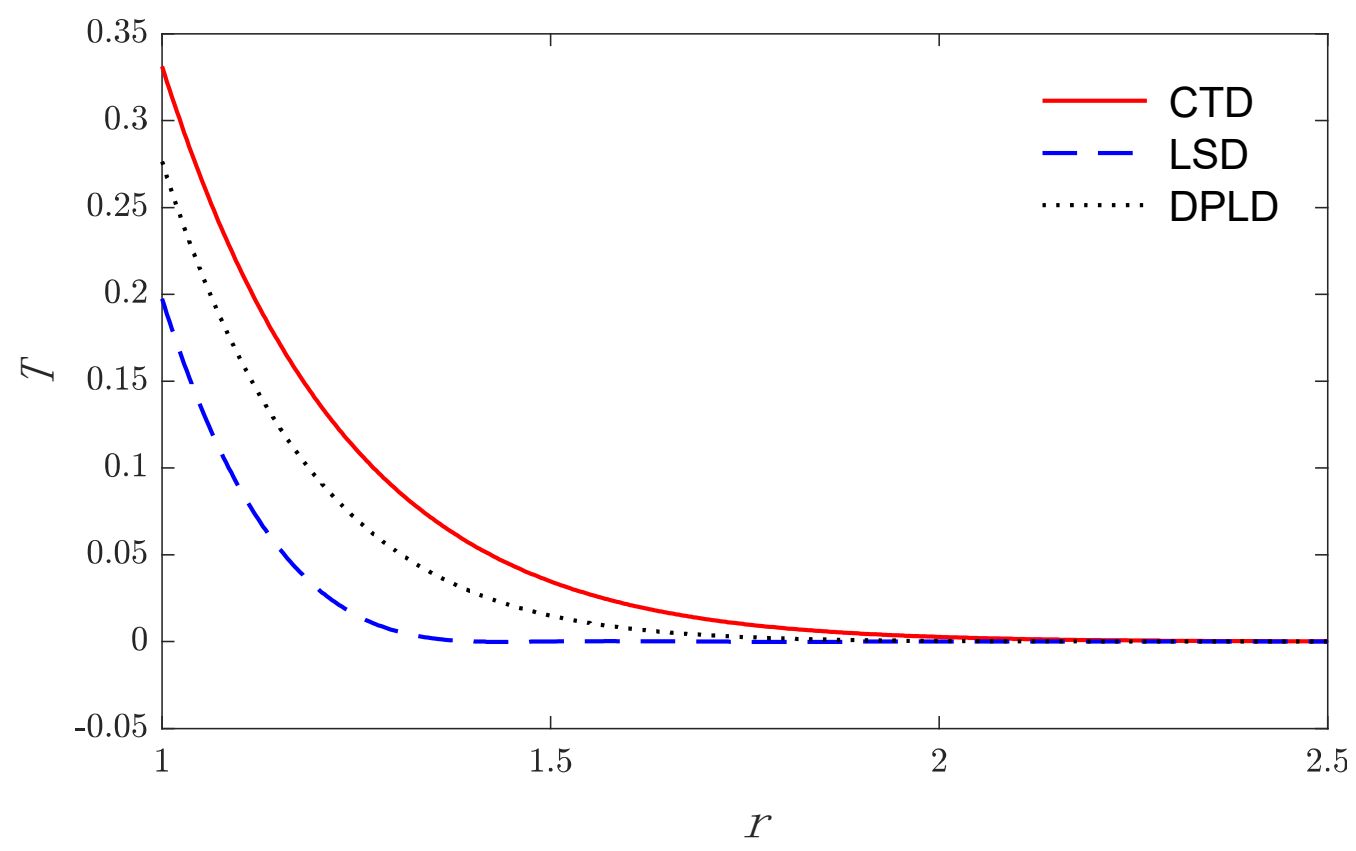

Figure 3. The temperature variations $T$ via the redial distance $r$ for three models. 


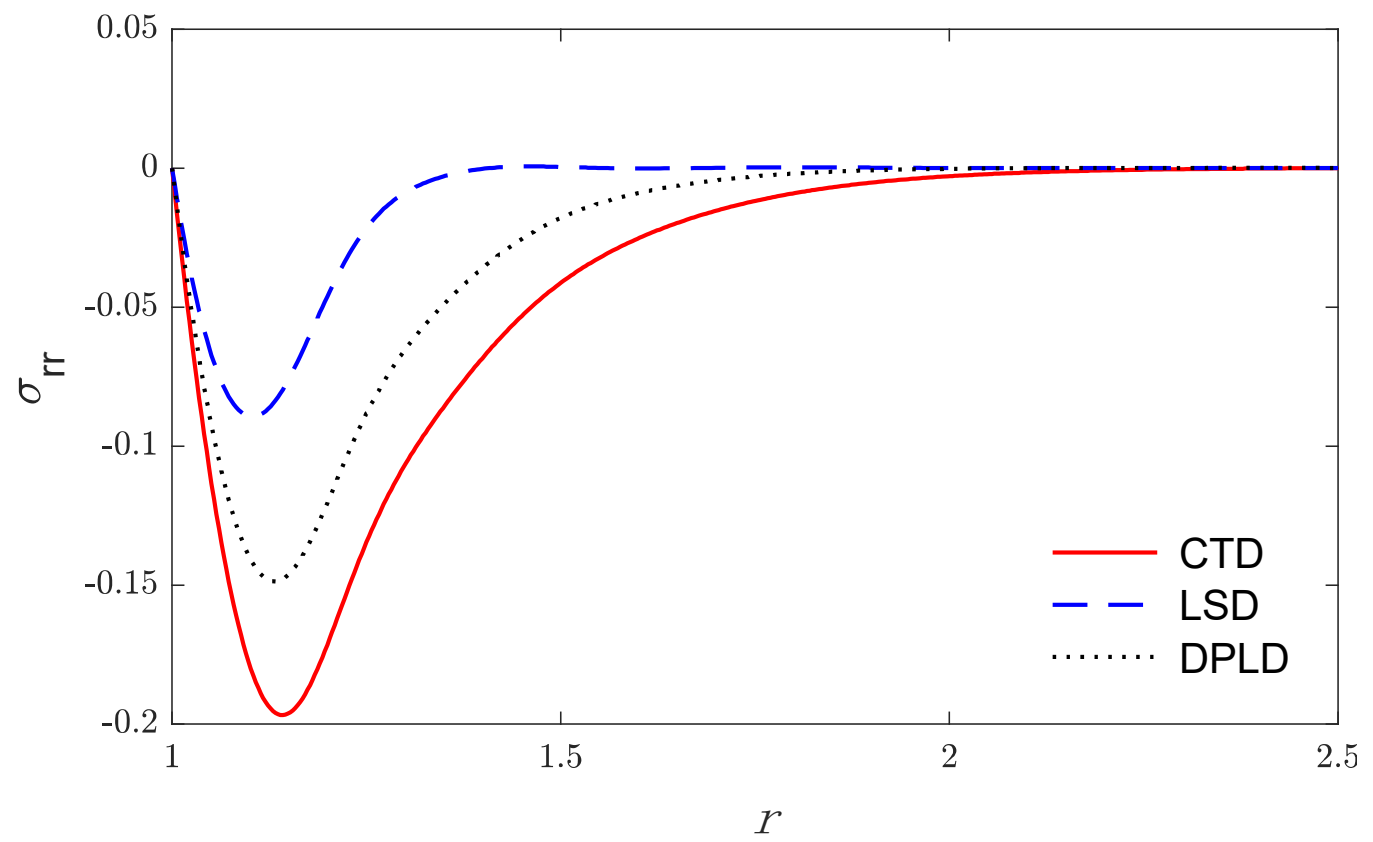

Figure 4. The redial stress variations $\sigma_{r r}$ along the redial distance $r$ for three models.

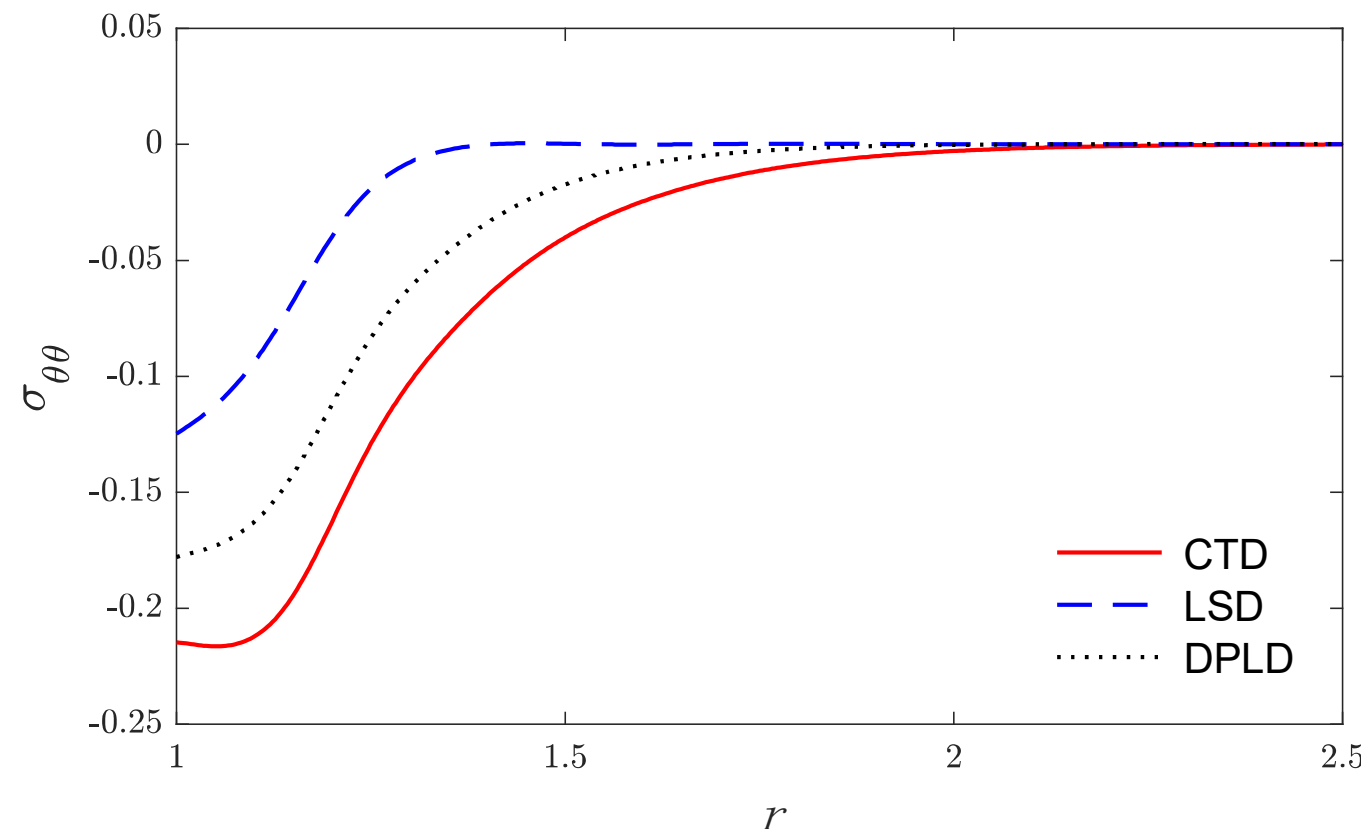

Figure 5. The shear stress variations $\sigma_{\partial \partial}$ versus the redial distance $r$ for three models. 


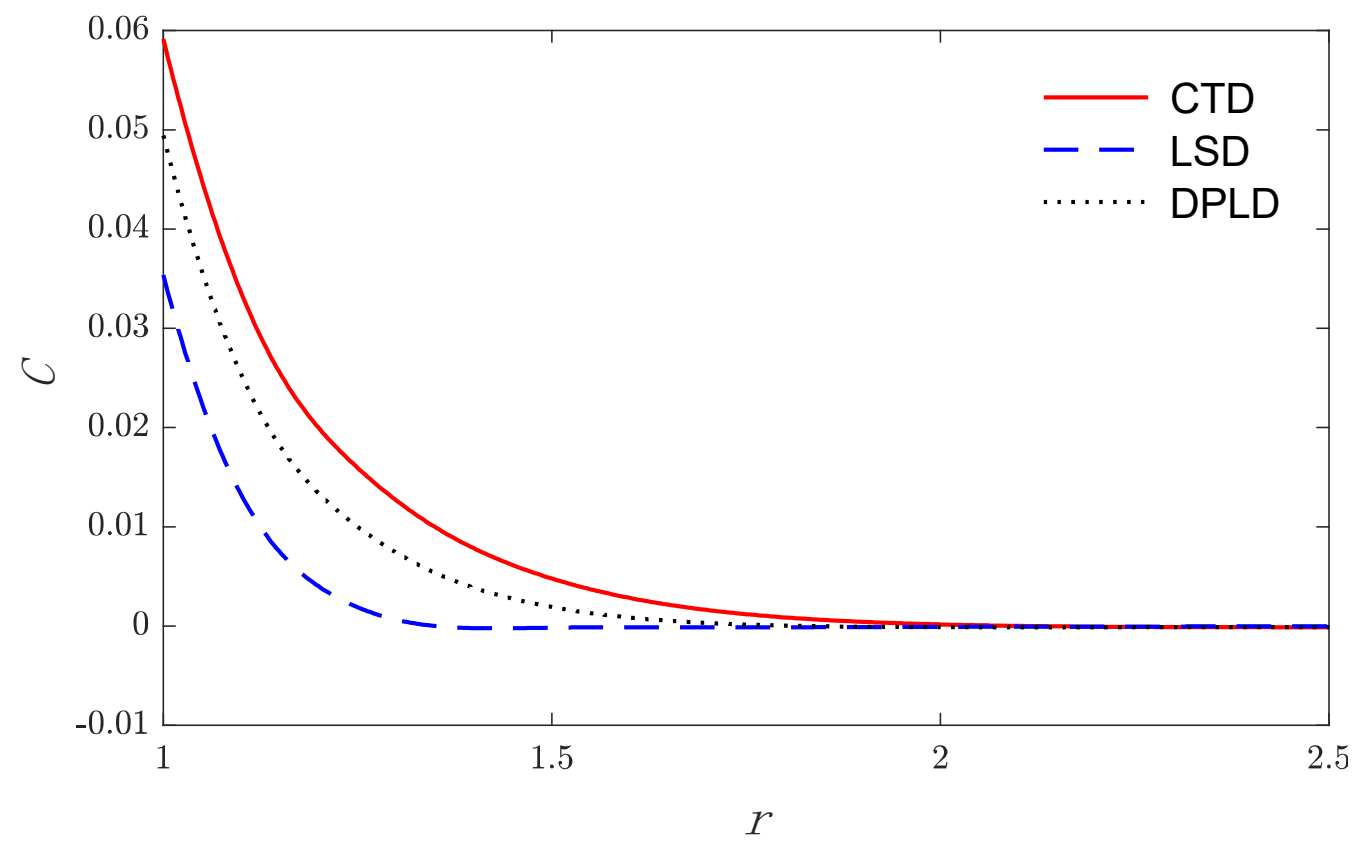

Figure 6. The concentration variation $C$ versus the redial distance $r$ for three models.

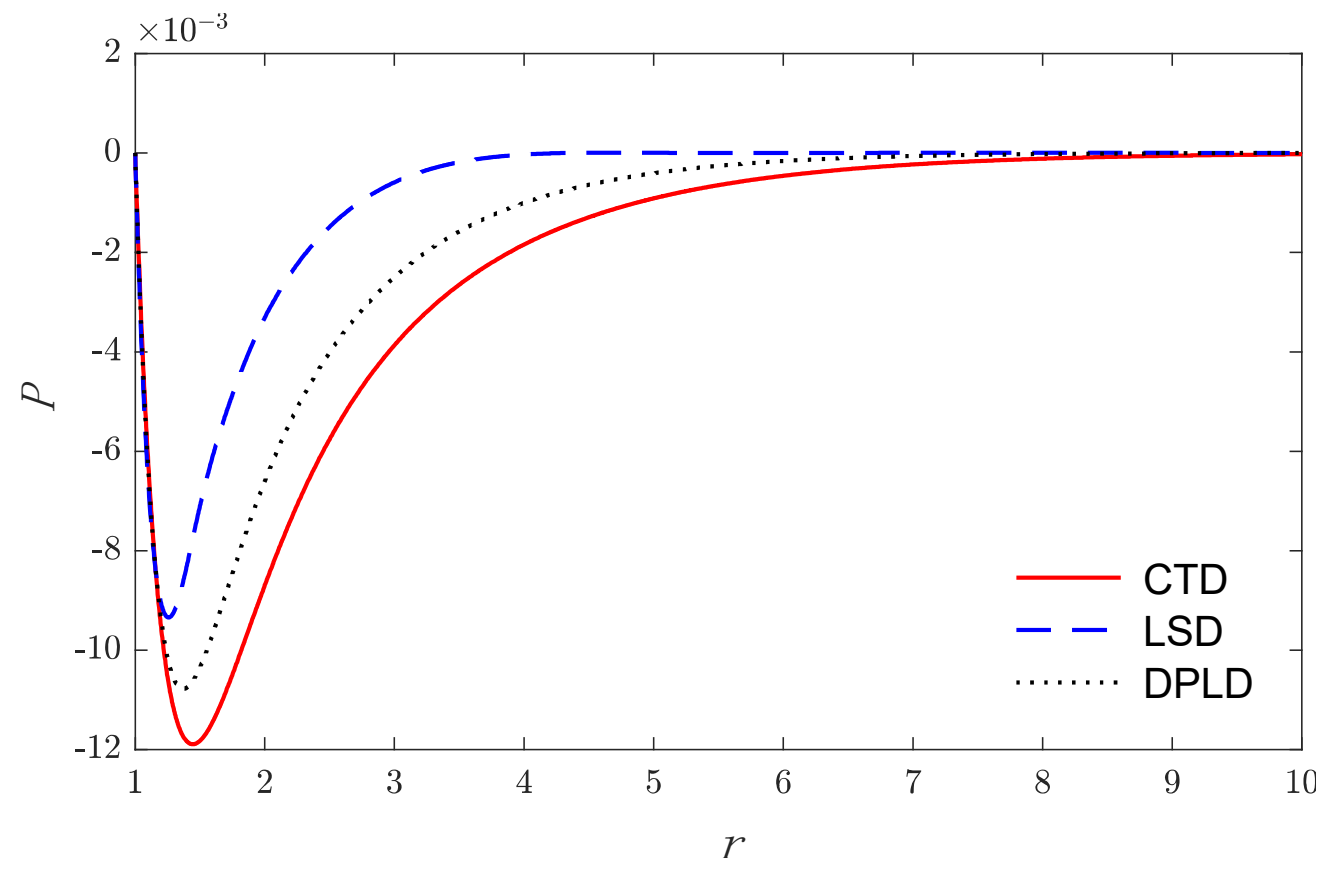

Figure 7. The chemical potential variations $P$ via the redial distance $r$ for three models.

\section{Conclusions}

This work aimed to mathematically analyze the effects of four thermal relaxation times. Based on the dual phase lag thermoelastic diffusion model, the radial displacement, the temperature change, the concentration, the chemical potential with the radial and the shear stresses distributions in the thermoelastic-diffusion medium have been presented. The finite element method has been used to obtain the numerical solutions for all physical quantities. The change in relaxation times (in the three models: DPLD, LSD and CTD) has significant effects and plays an important role in of the behavior of deformations of the various component of physical/chemical fields. Then, the parametric analysis has been completed to agree on the appropriate procedure to select important design 
variables to understand the basic characteristics of the new models of thermal conduction in thermoelastic-diffusion material.

Author Contributions: Conceptualization: A.D.H. and I.A.A.; methodology: A.D.H. and I.A.A.; validation: A.D.H. and I.A.A.; formal analysis: A.D.H. and I.A.A.; investigation: A.D.H. and I.A.A.; resources: I.A.A.; data curation: A.D.H. and I.A.A.; writing-original draft preparation: A.D.H. and I.A.A.; writing-review and editing: A.D.H.; visualization: I.A.A.; supervision: A.D.H. and I.A.A.; project administration: I.A.A. All authors have read and agreed to the published version of the manuscript.

Funding: The Deanship of Scientific Research (DSR) at King Abdulaziz University, Jeddah, Saudi Arabia has funded this project, under grant no. (FP-171-43).

Institutional Review Board Statement: Not applicable.

Informed Consent Statement: Not applicable.

Data Availability Statement: Not applicable.

Conflicts of Interest: The authors declare no conflict of interest.

\section{References}

1. Nowacki, W. Dynamic Problems of Thermoelasticity; Springer Science \& Business Media: Berlin/Heidelberg, Germany, 1975.

2. Biot, M.A. Thermoelasticity and irreversible thermodynamics. J. Appl. Phys. 1956, 27, 240-253. [CrossRef]

3. Lord, H.W.; Shulman, Y. A generalized dynamical theory of thermoelasticity. J. Mech. Phys. Solids 1967, 15, 299-309. [CrossRef]

4. Nowacki, W. Dynamic problems of diffusion in solids. Eng. Fract. Mech. 1976, 8, 261-266. [CrossRef]

5. Nowacki, W. Thermodiffusion in Solids. Mech. Teor. I Stosow. 1975, 13, 143-158.

6. Nowacki, W. Dynamical problem of thermodiffusion in solids 1. Bull. Acad. Pol. Sci. Ser. Sci. Tech. 1974, $22,55-64$.

7. Sherief, H.H.; Hamza, F.A.; Saleh, H.A. The theory of generalized thermoelastic diffusion. Int. J. Eng. Sci. 2004, 42, 591-608. [CrossRef]

8. Tzou, D.Y. Macro- to Micro-Scale Heat Transfer: The Lagging Behavior; Taylor \& Francis: Washington, DC, USA, 1996.

9. Abbas, I.A.; Singh, B. Finite Element Analysis in a Rotating Thermoelastic Half-Space with Diffusion. J. Comput. Theor. Nanosci. 2014, 11, 2276-2282. [CrossRef]

10. Villani, A.; Busso, E.P.; Ammar, K.; Forest, S.; Geers, M.G.D. A fully coupled diffusional-mechanical formulation: Numerical implementation, analytical validation, and effects of plasticity on equilibrium. Arch. Appl. Mech. 2014, 84, 1647-1664. [CrossRef]

11. Abbas, I.A.; Kumar, R.; Chawla, V. Response of thermal source in a transversely isotropic thermoelastic half-space with mass diffusion by using a finite element method. Chin. Phys. B 2012, 21, 084601. [CrossRef]

12. Sharma, J.N.; Kumari, N.; Sharma, K.K. Diffusion in a generalized thermoelastic solid in an infinite body with a cylindrical cavity. J. Appl. Mech. Tech. Phys. 2013, 54, 819-831. [CrossRef]

13. Abbas, I.A.; Marin, M. Analytical Solutions of a Two-Dimensional Generalized Thermoelastic Diffusions Problem Due to Laser Pulse. Iran. J. Sci. Technol. Trans. Mech. Eng. 2018, 42, 57-71. [CrossRef]

14. Abouelregal, A.E.; Elhagary, M.A.; Soleiman, A.; Khalil, K.M. Generalized thermoelastic-diffusion model with higher-order fractional time-derivatives and four-phase-lags. Mech. Based Des. Struct. Mach. 2020, 1-18. [CrossRef]

15. Sobhy, M. Hygrothermal vibration of orthotropic double-layered graphene sheets embedded in an elastic medium using the two-variable plate theory. Appl. Math. Model. 2016, 40, 85-99. [CrossRef]

16. Othman, M.I.; Eraki, E.E. Generalized magneto-thermoelastic half-space with diffusion under initial stress using three-phase-lag model. Mech. Based Des. Struct. Mach. 2017, 45, 145-159. [CrossRef]

17. Lotfy, K. Effect of variable thermal conductivity during the photothermal diffusion process of semiconductor medium. Silicon 2019, 11, 1863-1873. [CrossRef]

18. Kumar, R.; Sharma, N.; Lata, P. Effects of thermal and diffusion phase-lags in a plate with axisymmetric heat supply. Multidiscip. Model. Mater. Struct. 2016, 12, 275-290. [CrossRef]

19. Abbas, I.A.; Kumar, R. Deformation due to thermal source in micropolar generalized thermoelastic half-space by finite element method. J. Comput. Theor. Nanosci. 2014, 11, 185-190. [CrossRef]

20. Bezzina, S.; Zenkour, A.M. Thermoelastic diffusion of a solid cylinder in the context of modified Green-Naghdi models. Waves Random Complex Media 2021, 1-12. [CrossRef]

21. Madureira, R.L.R.; Rincon, M.A.; Aouadi, M. Numerical analysis for a thermoelastic diffusion problem in moving boundary. Math. Comput. Simul. 2021, 187, 630-655. [CrossRef]

22. Zenkour, A.M. Thermal diffusion of an unbounded solid with a spherical cavity via refined three-phase-lag Green-Naghdi models. Indian J. Phys. 2021, 1-18. [CrossRef]

23. Singh, B.; Yadav, A.K. The Effect of Diffusion on Propagation and Reflection of Waves in a Thermo-Microstretch Solid Half-Space. Comput. Math. Model. 2021, 32, 221-234. [CrossRef] 
24. Elhagary, M.A. Fractional thermoelastic diffusion problem for an infinitely long hollow cylinder using the Caputo-Fabrizio definition. J. Therm. Stresses 2021, 44, 281-294.

25. Molla, M.A.K.; Mallik, S.H. Variational principle, uniqueness and reciprocity theorems for higher order time-fractional fourphase-lag generalized thermoelastic diffusion model. Mech. Based Des. Struct. Mach. 2021, 1-16. [CrossRef]

26. Eremeyev, V.A.; Pietraszkiewicz, W. Nonlinear resultant theory of shells accounting for thermodiffusion. Contin. Mech. Thermodyn. 2021, 33, 893-909. [CrossRef]

27. Abbas, I.A. A dual phase lag model on thermoelastic interaction in an infinite fiber-reinforced anisotropic medium with a circular hole. Mech. Based Des. Struc. Mach. 2015, 43, 501-513. [CrossRef]

28. Saeed, T.; Abbas, I.; Marin, M. A GL Model on Thermo-Elastic Interaction in a Poroelastic Material Using Finite Element Method. Symmetry 2020, 12, 488. [CrossRef]

29. Marin, M.; Othman, M.I.A.; Seadawy, A.R.; Carstea, C. A domain of influence in the Moore-Gibson-Thompson theory of dipolar bodies. J. Taibah Univ. Sci. 2020, 14, 653-660. [CrossRef]

30. Marin, M. Some estimates on vibrations in thermoelasticity of dipolar bodies. JVC/J. Vib. Control. 2010, 16, 33-47. [CrossRef]

31. Zhang, L.; Zhang, X.M.; Zheng, H.W. Analysis on sintering driving force of unequal-sized particles in generalized thermoelastic diffusion. Fenmo Yejin Jishu/Powder Metall. Technol. 2019, 37, 259-263.

32. Shaw, S.; Othman, M.I.A. Computational analysis on the influence of damping in solid body deformation during thermoelastic mass diffusion. Waves Random Complex Media 2020, 1-21. [CrossRef]

33. Abo-dahab, S.M.; Abd-alla, A.M. Dual-phase-lag model on magneto-thermoelastic rotating medium with voids and diffusion under the effect of initial stress and gravity. Heat Transf. 2020, 49, 2131-2166. [CrossRef]

34. Othman, M.I.A.; Hilal, M.I.M.; Elmaklizi, Y.D. The effect of gravity and diffusion on micropolar thermoelasticity with temperaturedependent elastic medium under G-N theory. Mech. Mech. Eng. 2017, 21, 657-677.

35. Sharma, D.K.; Thakur, D.; Walia, V.; Sarkar, N. Free vibration analysis of a nonlocal thermoelastic hollow cylinder with diffusion. J. Therm. Stresses 2020, 43, 981-997. [CrossRef]

36. Alzahrani, F.S.; Abbas, I.A. Generalized thermoelastic diffusion in a nanoscale beam using eigenvalue approach. Acta Mech. 2016, 227, 955-968. [CrossRef]

37. Bajpai, A.; Sharma, P.K.; Kumar, R. Modeling of thermoelastic diffusion plate under two temperature, fractional-order, and temperature-dependent material properties. ZAMM Z. Fur Angew. Math. Und Mech. 2021, e202000321. [CrossRef]

38. Abbas, I.; Hobiny, A.D. Analytical-numerical solutions of photo-thermal interactions in semiconductor materials. Inf. Sci. Lett. 2021, 10, 189-196.

39. Bassiouny, E. Mathematical Model for Hyperbolic Two Temperature Fractional-Order Thermoelastic Materials Subjected to Thermal Loading. Appl. Math. Inf. Sci. 2021, 15, 23-29.

40. Abo-Dahab, S.M.; Abd-Alla, A.M.; Mahmoud, E.E. Thermal stresses in thermoelastic half-space without energy dissipation subjected to rotation and magnetic field. Appl. Math. Inf. Sci. 2017, 11, 1637-1647. [CrossRef]

41. Zenkour, A.M.; Abouelregal, A.E. Effect of temperature dependency on constrained orthotropic unbounded body with a cylindrical cavity due to pulse heat flux. J. Therm. Sci. Technol. 2015, 10, JTST0019. [CrossRef]

42. Abbas, I.A.; Youssef, H.M. A Nonlinear Generalized Thermoelasticity Model of Temperature-Dependent Materials Using Finite Element Method. Int. J. Thermophys. 2012, 33, 1302-1313. [CrossRef]

43. Abbas, I.A.; Othman, M.I.A. Generalized thermoelasticity of the thermal shock problem in an isotropic hollow cylinder and temperature dependent elastic moduli. Chin. Phys. B 2012, 21, 014601. [CrossRef]

44. Kumar, R.; Abbas, I.A. Deformation due to thermal source in micropolar thermoelastic media with thermal and conductive temperatures. J. Comput. Theor. Nanosci. 2013, 10, 2241-2247. [CrossRef]

45. Abbas, I.A.; Youssef, H.M. Finite element analysis of two-temperature generalized magneto-thermoelasticity. Arch. Appl. Mech. 2009, 79, 917-925. [CrossRef]

46. Sherief, H.H.; Saleh, H.A. A half-space problem in the theory of generalized thermoelastic diffusion. Int. J. Solids Struct. 2005, 42, 4484-4493. [CrossRef] 